\title{
CLINICAL RESEARCH ARTICLE Impact of a Make-A-Wish experience on healthcare utilization
}

\author{
Anup D. Patel ${ }^{1}$, Peter Glynn ${ }^{1}$, Ashley M. Falke ${ }^{1}$, Megan Reynolds ${ }^{1}$, Richard Hoyt ${ }^{1}$, Allison Hoynes ${ }^{1}$, Melissa Moore-Clingenpeel ${ }^{1}$,
} Ann Salvator ${ }^{1}$ and Jennifer J. Moreland ${ }^{1}$

\begin{abstract}
OBJECTIVES: To evaluate the impact of receiving a wish from the Make-A-Wish ${ }^{R}$ Foundation on (1) patient healthcare utilization and (2) savings benefit measures.

STUDY DESIGN: Make-A-Wish ${ }^{R}$ arranges experiences, or "wishes," to children with progressive, life-threatening, or life-limiting illness. A retrospective, case-control analysis was performed comparing patients who received or did not receive a wish and associated impact on healthcare utilization and costs across 2 years. Healthcare utilization was defined as visits to primary, urgent, emergent care, and planned/unplanned inpatient hospitalizations. We defined wish savings benefit as a decline in the cost of care from years 1 to 2, which exceeded the average cost of a wish in 2016, \$10,130.

RESULTS: From 2011 to 2016, 496 Nationwide Children's Hospital patients received a wish. We matched these patients to 496 controls based on age, gender, disease category, and disease complexity. Patients who received a wish were 2.5 and 1.9 times more likely to have fewer unplanned hospital admissions and emergency department visits, respectively. These decreases were associated with a higher likelihood (2.3-fold and 2.2-fold greater odds) of the wish achieving a savings benefit compared to hospital charges.

CONCLUSIONS: Participation in the Make-A-Wish ${ }^{R}$ program may provide children quality of life relief while reducing hospital visits and healthcare expenditures.
\end{abstract}

Pediatric Research (2019) 85:634-638; https://doi.org/10.1038/s41390-018-0207-5

\section{INTRODUCTION}

In the United States, chronic childhood illnesses doubled from $12.8 \%$ in 1994 to $26.6 \%$ in $2006 .{ }^{1}$ Chronic illnesses and conditions negatively impact patients' quality of life $^{2}$ and often require extensive and costly healthcare visits and procedures. ${ }^{3-5}$ Moreover, reimbursement is quickly moving from a fee-for-service to a value-based care model, resulting in an increase in the number of hospital groups and research teams designing interventions aimed at decreasing healthcare utilization for patients with chronic illness. ${ }^{6-8}$ Research demonstrates that children and their families can and do benefit from participation in interventions aimed at reducing the psychological and emotional strain associated with chronic illness management, ${ }^{9,10}$ and these interventions, in turn, can reduce healthcare costs.

The Make-A-Wish ${ }^{R}$ (MAW) Foundation is one such "intervention" offered to assist children and their families cope with and overcome illness. ${ }^{11}$ MAW is a 501 (c) ${ }^{3}$ non-profit organization whose goal is to provide children aged 3-17 years, with lifethreatening medical conditions, to experiences known as "wishes." Since 1980, MAW has granted over 415,000 wishes to children worldwide $^{11}$ dealing with either a progressive illness, a chronic life-limiting illness, or a life-threatening illness. The organization grants wishes based on qualifications in one of these three areas. For the progressive illness category, a child qualifies for a wish if the illness worsens or is expected to worsen over time. For the chronic life-limiting illness category, the child qualifies if the illness will limit or expected to limit their life expectancy significantly. Finally, the life-threatening illness category means the illness directly threatens the life of the child. A misconception may exist for who should be referred, as most children who are referred for a wish are not at the end of their life. Most recipients are referred for a wish by their medical provider. According to the MAW organization, the average national cost of a wish is $\$ 10,300$, which covers the expenses related to the wish experience and including overhead and staffing needs.

Schilling and Sarigiani et al. ${ }^{9}$ reported that benefits from a wish experience included feelings of positive anticipation prior to the wish, normalcy during the time of the chronic illness, lessened stress, and a decreased perception that the chronic illness impacted their lives post-wish. Similarly, results of a randomized controlled trial demonstrated significant quality of life improvements for those receiving a wish. ${ }^{10}$ While we know MAW is helpful in improving quality of life among pediatric patients with chronic illness, the literature currently lacks a robust evaluation of a wish receipt from the MAW Foundation as a resource and financially efficacious intervention. Therefore, we performed a retrospective analysis of children who received a wish from the MAW Foundation and measured the impact of wish receipt on the patient's subsequent healthcare utilization and related costs.

\section{METHODS}

After obtaining International Review Board approval from Nationwide Children's Hospital $(\mathrm{NCH})$, we conducted a retrospective cohort study of children with severe illnesses who received care at NCH facilities between 2011 and 2016. Children meeting the study criteria who had participated in the MAW program through $\mathrm{NCH}$ were matched with similar control children who had not received a wish experience.

${ }^{1}$ Department of Pediatrics and Neurology, Nationwide Children's Hospital, FOB 41.55, 700 Children's Drive, Columbus, OH 43205, USA

Correspondence: Ashley M. Falke (ashley.falke@nationwidechildrens.org)

Received: 8 June 2018 Revised: 22 August 2018 Accepted: 3 October 2018

Published online: 18 October 2018 
Context

$\mathrm{NCH}$ is a freestanding pediatric hospital with 427 licensed beds, 2 freestanding emergency departments (EDs), and 7 urgent care (UC) facilities. $\mathrm{NCH}$ is the sole tertiary pediatric care facility in Central Ohio. $\mathrm{NCH}$ clinicians partner with and refer patients and their families to the MAW program for a wish experience. Patients are eligible if they have a chronic illness with significant healthcare impact, a progressive illness, or a life-threatening illness. If a provider believes a patient qualifies for a wish, s/he can make a referral to the local or regional MAW chapter. Family members can also refer their child if they feel qualification exists based on using the same eligibility categories as described above. The regional chapter of MAW, Make-A-Wish Ohio, Kentucky, and Indiana, is the largest MAW chapter in the United States and grants the highest number of wishes per year.

\section{Sample derivation}

All MAW recipients were matched to a similar control to compare the potential effects of participation in the MAW program over a 2-year period. Data were extracted from patients' electronic health record (EHR). Using Structured Query Language (SQL) algorithms, we paired MAW patients with controls based, in order, on age categories, gender, disease category, and disease complexity. The age buckets were $2-7.998,8-12.998$, and 13 and above. Since children in the control group lacked a "wish date," we pulled healthcare utilization figures 1 year on either side of January 1, 2016; we verified control patients who visited the $\mathrm{NCH}$ after January 1, 2015 and prior to January 1, 2017 to ensure \pm 12 months of data surrounding our chosen control group "intervention" date. For the MAW participants, we extracted healthcare utilization data 1 year on either side of their wish date occurring between 2011 and 2016. Prior to matching, any patient who died prior to the end of the chosen 2-year period were excluded from analyses. To classify disease complexity for MAW and control patients, we employed the Pediatric Medical Complexity Algorithm to assign each patient a Simon Score. ${ }^{12-14}$ Traditionally, researchers sort patients into a 0 to 2 disease complexity category; ${ }^{12-14}$ however, we chose to use patients' raw and actual Simon Score to create a more robust intervention-to-control match.

Outcomes and analytic strategy

We extracted the following variables from patients' EHR: demographics (i.e., age, gender, insurance status); ED visits; UC visits; unplanned hospitalizations and associated length-of-stay (LOS); planned hospitalizations and associated LOS. In addition, we collected hospital charges for all healthcare utilization categories. An unplanned hospitalization was defined as an ED visit resulting in an inpatient hospitalization ${ }^{15}$ (Fig. 1). To ensure charges for services were "pure," we delineated, for instance, between ED visits and unplanned hospitalizations coming from ED visits.

We first compared the proportion of patients, across both wish conditions, whose ED, UC, and unplanned hospital admissions decreased from year 1 to year 2 using conditional logistic regression. Since 70\% year 1 and 79\% year 2 unplanned admissions, 5\% year 1 and 64\% year 2 ED visits, and $81 \%$ year 1 and $82 \%$ year 2 UC did not have a visit or admission, we first compared the proportion of patients whose unplanned visits decreased in year 2 using conditional logistic regression.

To assess the potential impact of MAW on charges, we created a new variable to indicate savings benefit, ${ }^{16}$ or whether each patient's charges were at least $\$ 10,130$ (i.e., the national average cost of a wish in 2016) less in year 2 compared to year 1 for healthcare utilization costs. We did not have data on the cost of each patient's actual wish. Conditional logistic regression was then used to compare the odds of achieving cost-effectiveness by MAW condition. All conditional logistic regression models were run first without covariates (unadjusted) and then with age and number of year 1 inpatient planned visits as covariates (adjusted). A subset of
MAW patients who had a unplanned or ED visits (year 1 or year 2) were analyzed for within-subject differences (MAW year 2 vs. year 1 and controls year 2 vs. year 1) and between-group differences (MAW year 2-year 1 difference vs. control year 2-year 1 difference) utilizing the sign test for non-parametric paired data (Table 5). The "savings benefit" variable was also calculated and compared between the matched MAW and control groups for unplanned and ED charges utilizing the McNemar test for paired data (Fig. 3). All analyses were conducted using SAS 9.4 (SAS Institute, Cary, NC, USA) and GraphPad Prism 7.03 (GraphPad Software, La Jolla, CA, USA).

\section{RESULTS}

Five hundred and sixty-eight patients at $\mathrm{NCH}$ received a wish between 2011 and 2016. Forty-five patients were excluded, as an adequate control utilizing the patients' cumulative Simon Score ${ }^{13,14}$ could not be found. An additional 27 patients were excluded due to death, which would limit our ability to accurately note healthcare utilization following a wish.

Four hundred and ninety-six MAW participants and 496 controls were matched on gender (48.6\% female for both groups), disease category (all $p>0.05$ ), and disease complexity ( $6.25 \pm 2.69$ vs. 5.99 $\pm 2.59)(p=0.002)$. The children in the MAW group had a higher complexity score. There were no statistically significant differences in health insurance coverage type at baseline (i.e., beginning of year 1) $(p>0.05)$. Children in the MAW condition were younger than matched controls $(9.85 \pm 4.94$ vs. $11.28 \pm 7.42, p=<0.0001)$ (Table 1).

On average, participants enrolled in the MAW program around 7 years of age $(M=7.30, S D=5.06)$ and received their wish by 10 years of age $(M=9.73, S D=4.91)$. Wishes received were classified

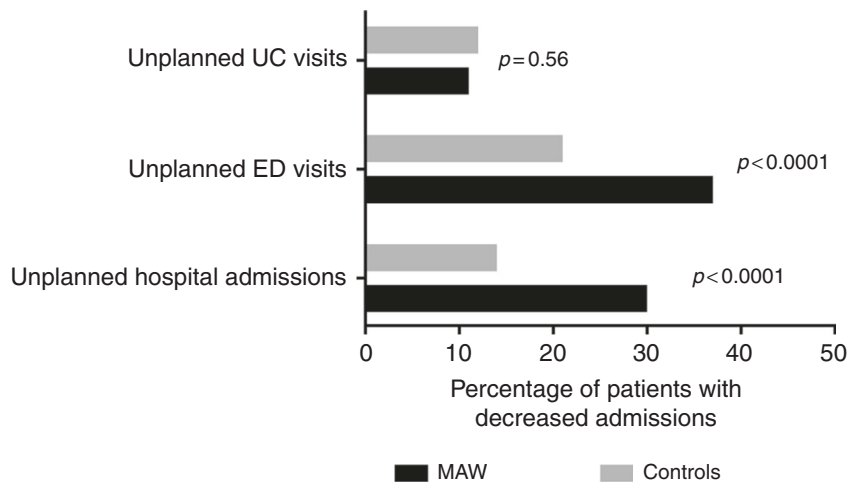

Fig. 1 Percentage of patients with decreased admissions

\begin{tabular}{|c|c|c|c|}
\hline Demographic characteristic & $\begin{array}{l}\text { MAW } \\
(N=496)\end{array}$ & $\begin{array}{l}\text { Control } \\
(N=496)\end{array}$ & $p$ value \\
\hline Female $(n(\%))$ & $241(48.6 \%)$ & $241(48.6 \%)$ & 1.00 \\
\hline $\begin{array}{l}\text { Age (years) at wish receipt (M } \\
\pm \mathrm{SD})\end{array}$ & $9.85 \pm 4.94$ & $11.28 \pm 7.42$ & 0.0004 \\
\hline \multicolumn{4}{|l|}{ Insurance type (pre-intervention) } \\
\hline 0 Self-pay/unlisted & $7(1.4 \%)$ & $12(2.4 \%)$ & 0.09 \\
\hline 1 Medicaid/government & $222(44.8 \%)$ & $255(51.4 \%)$ & \\
\hline 2 Private & $181(36.5 \%)$ & $155(31.2 \%)$ & \\
\hline $\begin{array}{l}3 \text { Both Medicaid/government } \\
\text { and private }\end{array}$ & $86(17.3 \%)$ & $74(14.9 \%)$ & \\
\hline Complexity score $(\mathrm{M} \pm \mathrm{SD})$ & $6.26 \pm 2.69$ & $5.99 \pm 2.59$ & 0.002 \\
\hline
\end{tabular}


Table 2. Wish choices

\begin{tabular}{|c|c|c|c|c|}
\hline & Type & MAW $(N=496)$ & Control $(N=496)$ & $p$ value \\
\hline \multicolumn{5}{|c|}{ Wish categories ${ }^{a}$} \\
\hline 1 & “Go” & $377(76.0 \%)$ & & \\
\hline 2 & "Meet" & $30(6.0 \%)$ & & \\
\hline 3 & "Have" & 87 (17.5\%) & & \\
\hline 4 & "Be" & $2(0.4 \%)$ & & \\
\hline \multicolumn{5}{|c|}{ Disease categories } \\
\hline 1 & Cancer/tumor & $222(44.8 \%)$ & $222(44.8 \%)$ & 1.00 \\
\hline 2 & Blood/renal & $82(16.5 \%)$ & $82(16.5 \%)$ & \\
\hline 3 & Neurologic & 72 (14.5\%) & 72 (14.5\%) & \\
\hline 4 & Genetic & 70 (14.1\%) & 70 (14.1\%) & \\
\hline 5 & Autoimmune & $5(1.0 \%)$ & $5(1.0 \%)$ & \\
\hline 6 & Heart & $42(8.5 \%)$ & $42(8.5 \%)$ & \\
\hline 7 & Lung & $1(0.2 \%)$ & $1(0.2 \%)$ & \\
\hline 8 & Unclassifiable & $2(0.4 \%)$ & $2(0.4 \%)$ & \\
\hline
\end{tabular}

per MAW Foundation's categories: "I wish to..." (a) "go" (i.e., destination selected) (76.0\%), (b) "be" (i.e., be someone for a day) (0.4\%), (c) "meet" (i.e., a famous individual) (6.0\%), and (d) "have" (i.e., receive a gift) (17.5\%). A majority of patients had a cancer (50.5\%) diagnosis, followed by a(n) blood/renal (14.4\%), neurologic (13.0\%), genetic (12.7\%), heart (7.7\%), or "other" (i.e., autoimmune, lung, unclassifiable) (2.2\%) diagnosis (Table 2 ).

After accounting for age and year 1 planned inpatient visits, patients who received a wish were 2.5 and 1.9 times more likely to experience a drop in unplanned hospital admissions and ED visits, respectively $(p<0.0001)$, compared to patients who did not receive a wish. Furthermore, when accounting for age and year 1 planned inpatient visits, children who receive a wish were 2.25 and 2.18 times more likely to have utilization costs achieve savings benefit for unplanned admission and ED charges, respectively $(p<0.0001)$, compared to control children. Children in the MAW group were not less likely than control children to visit the UC post intervention (Tables 2 and 3).

When comparing healthcare charges from year to year for ED visits and after accounting for the cost of a wish, 30\% of the wish children were determined to have savings benefit vs. $15 \%$ in the control group ( $p=0.0001)$. Similar results were seen in regards to unplanned hospitalizations (Fig. 2). When evaluating changes in hospital charges, $62 \%$ of patients in the wish group had less charges in the year 2 compared to year 1 , with $42 \%$ of patients in the control group $(p=0.02)$. A statistical significant difference was not seen in regards to ED charges (Fig. 3).

A subset analysis was performed among case-control pairs where both the control patient and the patient who received a wish had at least one unplanned or ED visit $(N=154)$ (Table 4$)$, in order to focus on patients who had at least some healthcare utilization during the study period. In this subset, patients who received a wish had a statistically significant decline in unplanned visits (MAW year 2 -year 1 difference of $-0.84 \pm 1.42$ visits, $p<$ $0.0001)$, while patients in the control group had a slight increase in the number of visits $(0.18 \pm 2.14, p>0.05)$ (Table 5).

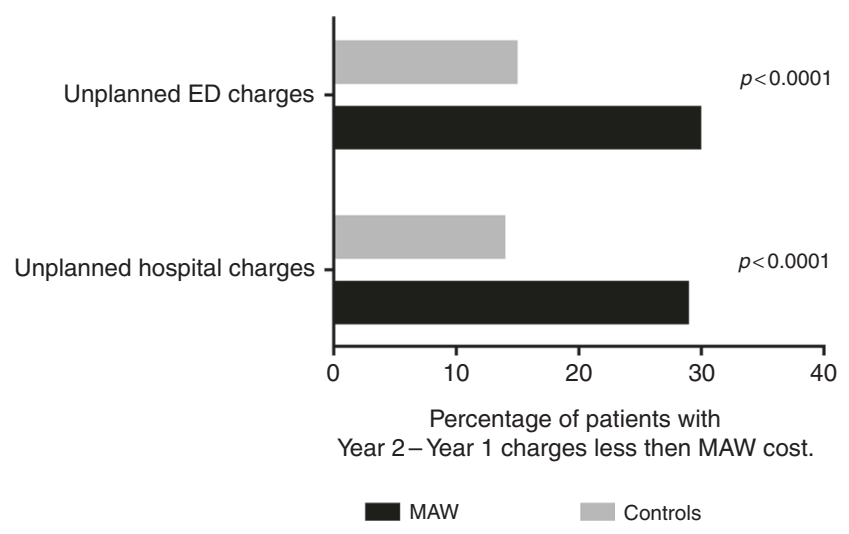

Fig. 2 Percentage of patients with year 2-year 1 charges less than MAW cost

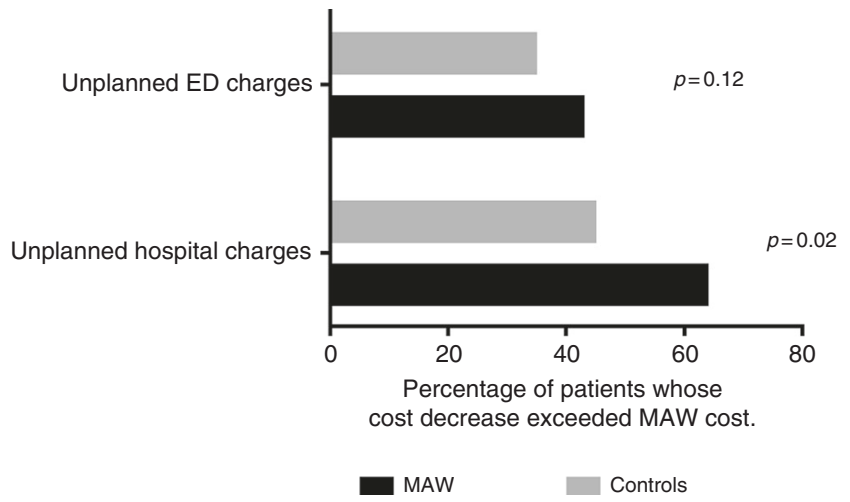

Fig. 3 Percentage of patients with year 2-year 1 charges less than MAW cost

\section{DISCUSSION}

The goal of this retrospective, chart review study was to statistically measure and validate any improvement in the quality of life of children with life-threatening medical conditions, as well as the return on investment (ROI) for the MAW Foundation by evaluating healthcare utilization and costs following a wish. We employed a rigorous matching algorithm ${ }^{13,14}$ to ensure the validity of our results and confirm that our control group was similar to children who received a wish with regard to gender, disease, and disease complexity. The choices for wishes made by the patients in our study mirror similar published data, ${ }^{9}$ thus reflecting similarities among our population compared to other wish populations.

Compared to the control group, children receiving a wish were more likely to experience decreases in unplanned hospitalizations, ED visits, and hospital charges. In this study, we defined savings benefit as cost savings equal or more to that of the national average of a wish, ${ }^{11}$ or $\$ 10,130$. A higher percentage of wish kids achieved a decrease in healthcare costs compared to those children who did not receive a wish.

Our study is the first to formally assess a nationally recognized child advocacy program on healthcare utilization and cost metrics. Other metrics have been evaluated previously. A past study demonstrated programs similar to the MAW experience aid in improving quality of life and family bonding, increasing hope, reducing stress, and functioning as a distraction from illness. 910 Results of other studies showed internalized hope can improve patient behavior, such as treatment adherence. ${ }^{17,18}$ Further, stress 


\begin{tabular}{|c|c|c|c|c|}
\hline \multicolumn{5}{|l|}{ Unplanned admissions } \\
\hline MAW vs. control & $2.65(1.89,3.69)$ & $<0.0001$ & $2.48(1.74,3.50)$ & $<0.0001$ \\
\hline Pre-planned visits year 1 & $1.07(1.02,1.11)$ & 0.005 & $1.07(1.02,1.12)$ & 0.005 \\
\hline Age (years) & $0.87(0.78,0.96)$ & 0.009 & $0.86(0.77,0.97)$ & 0.01 \\
\hline \multicolumn{5}{|l|}{ ED visits } \\
\hline Pre-planned visits pre year 1 & $1.05(1.01,1.09)$ & 0.02 & $1.05(1.01,1.09)$ & 0.02 \\
\hline Age (years) & $0.92(0.84,0.99)$ & 0.03 & $0.91(0.84,0.99)$ & 0.03 \\
\hline \multicolumn{5}{|l|}{ UC visits } \\
\hline MAW vs. control & $0.89(0.61,1.31)$ & 0.56 & $0.88(0.59,1.31)$ & 0.53 \\
\hline Pre-planned visits year 1 & $1.01(0.96,1.07)$ & 0.75 & $1.01(0.95,1.06)$ & 0.78 \\
\hline
\end{tabular}

\begin{tabular}{|c|c|c|c|c|}
\hline MAW vs. control & $2.55(1.82,3.58)$ & $<0.0001$ & $2.25(1.55,3.25)$ & $<0.0001$ \\
\hline Pre-planned visits year 1 & $2.46(1.73,3.54)$ & 0.0006 & $1.09(1.04,1.15)$ & 0.0009 \\
\hline Age (years) & $0.85(0.76,0.95)$ & 0.004 & $0.85(0.75,0.96)$ & 0.008 \\
\hline Pre-planned visits pre year 1 & $1.09(1.04,1.15)$ & 0.0009 & $1.09(1.03,1.14)$ & 0.001 \\
\hline Age (years) & $0.85(0.77,0.95)$ & 0.004 & $0.85(0.76,0.96)$ & 0.007 \\
\hline
\end{tabular}

Table 5. Differences in charges (year 2-year 1) for MAW patients who had an unplanned or ED visit matched to their controls

\begin{tabular}{lll}
\hline & MAW $(N=77)$, year 2-year 1 & Controls $(N=77)$, year 2-year 1 \\
\hline Unplanned visits & $-0.84 \pm 1.42^{\mathrm{a}}$ & $0.18 \pm 2.14$ \\
Unplanned visits LOS & $-4.30 \pm 12.05^{\mathrm{b}}$ & $-5.35 \pm 26.54$ \\
Unplanned charges & $-35,181.19 \pm 108,686.91^{\mathrm{c}}$ & $-70126.71 \pm 368,700.80$ \\
& MAW $(N=159)$, year 2-year 1 & Controls $(N=159)$, year 2-year 1 \\
ED visits & $-0.33 \pm 2.91$ & $-0.14 \pm 2.64$ \\
ED charges & $-19,227.33 \pm 210,452.90^{\mathrm{d}}$ & $-49,271.49 \pm 263,605.21^{\mathrm{e}}$ \\
\hline a The MAW group had a statistically significant decrease (year 2-year 1) in unplanned visits $(p<0.0001)$ & \\
bThe MAW group had a statistically significant decrease (year 2-year 1) in unplanned visits LOS $(p<0.0001)$ & \\
c The MAW group had a statistically significant decrease (year 2-year 1) in unplanned visits charges $(p<0.0001)$ & \\
d The MAW group had a statistically significant decrease (year 2-year 1) in ED charges $(p=0.007)$ & \\
e The Control group had a statistically significant decrease (year 2-year 1) in ED charges $(p=0.01)$ & \\
\hline
\end{tabular}

reduction and a reduction in psychological symptoms such as depression and anxiety were noted in a study evaluating children with oncological illness compared to those on a wait list for a wish. ${ }^{10}$ Distraction from illness ${ }^{19}$ and family bonding has shown to have positive correlation on improved quality of life for patients and their families with chronic medical illness. ${ }^{9,19-21}$ Such findings may explain the reduction in healthcare utilization and associated costs and may serve as explanations on the results we note.

Our study is limited by factors common to retrospective chart review studies. First, events outside the wish "intervention" may have affected outcomes. Further, we were not able to capture visits occurring outside the $\mathrm{NCH}$ network, as they are not recorded in our EHR. However, historically, most of the children with similar chronic illness receive all their care from our hospital and the same limitations existed in our wish and control populations. ${ }^{22}$ Importantly, data from our study comes from a single site, thus our findings may not represent other geographic regions in the United States. The children in our study who received a wish were younger; therefore, it is possible members of this group were viewed as more resilient and/or sicker. However, we employed an additional level of rigor using complexity ${ }^{13,14}$ matching to help equalize our intervention and control groups.

In summary, children who received a wish experience from the MAW Organization were more likely to experience significant decreases in healthcare utilization and costs, across 2 years, compared to similar children who did not receive a wish. Investing 
in wish granting may aid in improved quality of life of these children and in controlling healthcare costs by providing a valuable $\mathrm{ROI}$ to the healthcare system. Based on our findings, insurance providers may be interested in assuring eligible children receive a wish to further help cost control.

The fact the control group did not receive a wish may represent a gap in the ability to provide a wish to all eligible children. Unfortunately, we do not know if these patients were offered a wish and declined vs. never referred to the MAW. Our analysis did not evaluate factors associated with not receiving a wish; however, the common misconception that a child must be nearing end of life likely plays a role in children not receiving a wish when eligible. Further work to validate wish granting criteria with the EHR may help create tools to identify those children who may be eligible for a wish, thus aiding MAW America and their goal of providing every eligible child a wish experience. ${ }^{11}$ Knowing that our results demonstrate a decrease in healthcare utilization and cost savings, donors may be more likely to donate to MAW America for their goal to be realized.

Further work on the specific benefits would be helpful to gain greater understanding as to the reasons behind these findings. A similar study evaluating multiple centers in various regions of the United States would be helpful to validate our findings. Ideally, a randomized, controlled trial would be helpful to substantiate our findings; however, such a study may not be ethical given benefits already demonstrated for children who receive a wish experience. ${ }^{10}$ In conclusion, our study demonstrates that a wish from MAW America can have profound impacts from improved quality of life ${ }^{10}$ to a reduction in healthcare utilization.

\section{AUTHOR CONTRIBUTIONS}

A.D.P. conceptualized and designed the study and wrote the initial draft of the manuscript. P.G. conceptualized and designed the study and assisted in writing the initial draft of the manuscript. J.J.M. designed the study, aided in analyzing data, and wrote portions of the initial draft. M.R. and R.H. created the SQL code used to abstract data and assisted in writing the initial draft of the manuscript. A.H. conceptualized and designed the study and performed data collection. M.M.-C. and A.S. designed the study and analyzed study data. All authors edited and approved the final manuscript as written and take responsibility for its content.

\section{ADDITIONAL INFORMATION}

Competing interests: A.D.P. volunteers as a member of the Medical Advisory Council for MAW America. A.D.P. wrote the first draft of this manuscript. The other authors declare no competing interests.

Publisher's note: Springer Nature remains neutral with regard to jurisdictional claims in published maps and institutional affiliations.

\section{REFERENCES}

1. Van Cleave, J., Gortmaker, S. L. \& Perrin, J. M. Dynamics of obesity and chronic health conditions among children and youth. JAMA 303, 623-630 (2010).

2. Thomson, J. et al. Financial and social hardships in families of children with medical complexity. J. Pediatr. 172, 187-193 (2016).

3. Agrawal, R. et al. Trends in health care spending for children in medicaid with high resource use. Pediatrics 4, 138 (2016).

4. Berry, J. G. et al. Impact of chronic conditions on emergency department visits of children using medicaid. J. Pediatr. 182, 267-274 (2017).

5. Widger, K., Seow, H., Rapoport, A., Chalifoux, M. \& Tanuseputro, P. Children's endof-life health care use and cost. Pediatrics 4, 139 (2017).

6. Clough, J. D. \& McClellan, M. Implementing MACRA: implications for physicians and for physician leadership. JAMA 315, 2397-2398 (2016).

7. Mullins, A. Medicare payment reform: making sense of MACRA. Fam. Pract. Manag. 23, 12-15 (2016).

8. Mulvany, C. MACRA: the Medicare physician payment system continues to evolve. Healthc. Financ. Manag. 70, 32-35 (2016)

9. Schilling, M. L. \& Sarigiani, P. The impact of a wish: caregiver perceptions of the benefits of granted wishes for children with life-threatening illnesses and their families. Children's Health Care 43, 16-38 (2014).

10. Shoshani, A., Mifano, K. \& Czamanski-Cohen, J. The effects of the Make a Wish intervention on psychiatric symptoms and health-related quality of life of children with cancer: a randomised controlled trial. Qual. Life Res. 25, 1209-1218 (2016).

11. Make A Wish America. 2017. Accessed November 21, 2017 http://wish.org/.

12. O'Mahony, L. et al. Medical complexity and pediatric emergency department and inpatient utilization. Pediatrics 131, e559-e565 (2013).

13. Simon, T. D. et al. Pediatric medical complexity algorithm: a new method to stratify children by medical complexity. Pediatrics 133, e1647-e1654 (2014).

14. Simon, T. D., Cawthon, M. L., Popalisky, J. \& Mangione-Smith, R. Development and Validation of the Pediatric Medical Complexity Algorithm (PMCA) Version 2.0. Hosp. Pediatr. 7, 373-377 (2017).

15. Patel, A. D., Wood, E. G. \& Cohen, D. M. Reduced emergency department utilization by patients with epilepsy using QI methodology. Pediatrics. 2, 139 (2017).

16. Ekwaru, J. P. et al. Cost-effectiveness of a school-based health promotion program in Canada: a life-course modeling approach. PLOS ONE 12, e0177848 (2017).

17. Berg, C. J., Rapoff, M. A., Snyder, C. R. \& Belmont, J. M. The relationship of children's hope to pediatric asthma treatment adherence. J. Posit. Psychol. 2, 176-184 (2007).

18. Santos, S., Crespo, C., Canavarro, M. C. \& Kazak, A. E. Family rituals and quality of life in children with cancer and their parents: the role of family cohesion and hope. J. Pediatr. Psychol. 40, 664-671 (2015).

19. Kato, P. M., Cole, S. W., Bradlyn, A. S. \& Pollock, B. H. A video game improves behavioral outcomes in adolescents and young adults with cancer: a randomized trial. Pediatrics 122, e305-e317 (2008).

20. Galinsky, J. What do families, staff, and stakeholders think about children's dream fulfilment? Issues Compr. Pediatr. Nurs. 37, 203-211 (2014).

21. Darlington, A. S., Heule, F. \& Passchier, J. Granting wishes: parents' perception of a wish fulfilment for a child with a life-threatening illness. Acta Paediatr. 102, e480-e482 (2013).

22. Kelleher, K. J. et al. Cost saving and quality of care in a pediatric accountable care organization. Pediatrics 135, e582-e589 (2015). 